\title{
Modélisation mathématique à correction d'erreurs de la liquidité du marché boursier. Application : Impact de la structure du marché et du drainage de l'épargne institutionnelle sur le marché des capitaux
}

\section{Mathematical error-correction modeling of the liquidity of the stock market. Application: Impact of Market Structure and Institutional Savings on the Capital Market}

\author{
Mustapha Bouchekourte ${ }^{1}$, Norelislam El Hami ${ }^{2}$ \\ ${ }^{1}$ Laboratoire Sciences de Gestion, Univérsité Hassan II - Casablanca, Maroc, bouchekourte@depf.finances.gov.ma \\ ${ }^{2}$ Laboratoire Génie des Systèmes (LGS), ENSA-Kénitra, Université Ibn Tofail, Maroc, norelislam@outlook.com
}

RÉSUMÉ. Un modèle mathématique est estimé à partir des variables significatives (après l'élimination des variables peu influentes) et à partir des variables dummy introduites pour corriger quelques évènements conjoncturels qui impactent les corrélations ordinaires des variables. II peut exister plusieurs combinaisons linéaires stationnaires entre des variables intégrées d'ordre un. Dans la méthode de Johansen, la détermination de la dimension de l'espace de cointégration se fait par l'estimation d'un modèle autorégressif.

Nous utiliserons le ratio de liquidité comme variable proxy de la liquidité du marché boursier marocain, pour estimer les indicateurs et les facteurs qui déterminent sa variabilité à court et à long terme. La méthode économétrique appropriée, serait d'estimer un modèle vectoriel à correction d'erreur (VECM) qui a la propriété de déterminer les relations de long et de court terme entre les variables.

ABSTRACT. A mathematical model is estimated from the significant variables (after the elimination of the low influencing variables) and from dummy variables introduced to correct some cyclical events that impact the ordinary correlations of the variables. There may be several stationary linear combinations between built-in variables of order one. In Johansen's method, the cointegration space is determined by estimating an autoregressive model.

We will use the liquidity ratio as a proxy for the liquidity of the Moroccan stock market, to estimate the indicators and the factors that determine its variability in the short and long run. The appropriate econometric method would be to estimate a vector error correction model (VECM) that has the property of determining the long and short term relationships between variables.

MOTS-CLÉS. Modèle vectoriel, cointégration, ratio de liquidité, Bourse de Casablanca.

KEYWORDS. Vector model, cointegration, liquidity ratio, Casablanca Stock Exchange.

\section{Introduction}

L'hypothèse d'avoir un facteur commun de la liquidité consiste à supposer que lors d'un choc de liquidité, tout le marché sera affecté dans son ensemble [1].

La liquidité aurait par conséquent deux composantes : la première est spécifique représentant ses déterminants liés discrètement aux titres cotés et une deuxième systématique intégrant des caractéristiques communes à tous les titres et liées aux marchés [2]. On distingue généralement deux catégories de facteurs à savoir les facteurs macroéconomiques et les facteurs structurels propres aux aléas du marché boursier. 


\section{Facteurs macroéconomiques}

Les modèles basés sur le risque de stockage de Demsetz (1968), Stoll (1978), Ho et Stoll (1981) et Biais(1993) affirment que le niveau de liquidité est lié au stockage des titres. Le coût de détention des actions est assimilé aux intérêts rapportés par le placement de la valeur en monnaie des titres détenus. Par conséquent, la liquidité des titres dépend indirectement du niveau des taux d'intérêt à court et à long terme, représenté par le marché obligataire et celui des bons de trésor. Les actifs produits sur ces marchés constituent une alternative aux actions cotées jugés cycliquement comme moins rentable. De même, les prix de l'immobilier sont considérés comme étant des valeurs refuges si les gains réalisés sur les marchés boursiers sont minimes.

\section{Facteurs structurels}

Les variables liées à l'organisation et à l'activité du marché peuvent expliquer également les variations communes de liquidité. Elles regroupent le volume, le nombre de transactions, le nombre de contrats négociés, la volatilité de l'indice, le flottant et la capitalisation boursière du marché. Elles concernent des déterminants spécifiques de liquidité qui peuvent agir seules ou en interaction avec d'autres facteurs pour influencer la liquidité. De plus une forte rentabilité sur le marché entraine un attrait plus fort de la part des investisseurs. Ceci conduit à un accroissement de l'activité [3], et par conséquent une modification des paramètres de liquidité des titres composant 1'indice de marché.

\section{Données et méthodologie}

Les données utilisées dans cette analyse proviennent des sources nationales (ACAPS, caisses de retraite, assurances, ASFIM, Ministère des finances, Bank AL-Maghrib, AMMC et Bourse de Casablanca). Elles couvrent la période 2004-2014. L'analyse porte sur plusieurs variables macroéconomiques et de microstructure.

La liquidité est une caractéristique décisive pour les investisseurs car elle est - associée au rendement - le premier critère d'arbitrage entre les placements [4]. Nous allons essayer de comprendre son origine, d'identifier ses causes et d'explorer le modèle sa variabilité en vue d'apprécier l'impact des placements sur son évolution. Notre approche a pour objectif d'intégrer dans l'analyse de la liquidité la dimension de la gestion des placements en repérant à chaque fois les liaisons de la liquidité aux différentes pratiques et choix de gestion [5]. 


\begin{tabular}{|l|l|}
\hline Ratio de liquidité & RL \\
\hline Courbe des taux du marché secondaire de 10 ans & CTM10 \\
\hline Courbe des taux du marché secondaire de 13 semaines & CTM13 \\
\hline Courbe des taux du marché secondaire de 52 semaines & CTM52S \\
\hline Le taux d'inflation & INFLATION \\
\hline Logarithme de l'agrégat M1 & LNM1 \\
\hline Logarithme de l'agrégat M3 & LNM3 \\
\hline L'indice MASI & MASI \\
\hline Nombre de contrats sur le marché de bloc & NCMB \\
\hline Nombre de contrats sur le marché central & NCMC \\
\hline OPCVM obligataires et monétaire à long terme & OMLT \\
\hline Actif net des OPCVM actions & OPCVM_ACTIONS \\
\hline Actif net des OPCVM monétaires & OPCVM_MONETAIRE \\
\hline Part des actions de l'actif net total des OPCVM & PART \\
\hline Volatilité du MADEX & VOLMADEX \\
\hline Volatilité du MASI & VOLMASI \\
\hline Volume total de transactions & VTMCDIVM \\
\hline
\end{tabular}

\section{Codification des variables estimés}

\section{L'analyse économétrique de la relation entre le ratio de liquidité et paramètres du marché des capitaux}

Nous prenons la variable RL représentant le ratio de liquidité comme variable proxy de la liquidité $\mathrm{du}$ marché boursier marocain. L'analyse porte sur plusieurs variables macroéconomiques et de microstructure.

La méthode économétrique appropriée [6], serait d'estimer un modèle vectoriel à correction d'erreur (VECM) qui a la propriété de déterminer les relations de long et de court terme entre les variables.

Une des étapes les plus importantes qui précède le test de cointégration multivarié de Johansen est la recherche du nombre de retards optimal. Le choix du nombre de retards peut affecter sensiblement les résultats des tests 1 . Si le nombre de retards est insuffisant, le modèle peut retenir de l'autocorrélation au sein de son terme résiduel, et si, par contre, l'ordre du VAR est trop grand, les tests tendent à surestimer le nombre de relations de cointégration. Le nombre de retards est déterminé à partir des critères d'information usuels tels que les critères d'Akaike et Schwartz et Hannan-Quinn [7]. Ces critères s'appuient sur l'apport d'information généré par des retards supplémentaires dans le modèle.

Notre modèle est estimé à partir des variables significatives (après l'élimination des variables non significatives) et à partir des variables dummy [8] introduites pour corriger quelques évènements conjoncturels qui ont influencé les corrélations ordinaires des variables. Le modèle est présenté ainsi :

\footnotetext{
${ }^{1}$ En effet les travaux de Boswijk et Fances (1992), Gonzalo (1994) et Ho et Sorensen (1996) ont bien souligné ce constat. 
$D(R L)=C(1)+C(2) * D(V O L M A S I)+C(3) * D(N C M C)+C(4) * R L(-1)+C(5) * L O G(M 3(-1))+C(6) * L O G(M A S I(-1))+C(7) * C T M 52 S(-1)+$ C(8)*PART(-1) + C(9)*NCMC(-1) + C(10)*VOLMASI(-1) + C(11)*DUM0409 + (12)*DUM0812 + C(13)*DUM0504 + C(14)*DUM0512 + $C(15) * D U M 0612^{2}+[A R(12)=C(16), E S T S M P L=" 2004 M 02$ 2014M12"']

\section{GARCH $=\mathrm{C}(17)+\mathrm{C}(18)^{*} \mathrm{RESID}(-1)^{\wedge} 2+\mathrm{C}(19)^{*} \mathrm{GARCH}(-1)$}

Lorsque les variables sont intégrées d'ordre 1 et cointégrées (Engle-Granger 1987, Granger 1988, Johanson 1988) il faut recourir aux modèles à correction d'erreurs (VECM) permettant de tester la causalité sur les modèles à court et long terme. Celle de court terme étant obtenue à partir des coefficients associés aux variables explicatives différenciées tandis que la causalité de long terme provient des variables utilisées dans le vecteur de cointégration. Cependant, au regard des limites et de la faiblesse des tests de racine unitaire pour les échantillons de petite taille, les tests de cointégartion de Johanson ont tendance à rejeter l'hypothèse d'absence de cointégration (Toda et Yamamoto, 1995) en relation notamment avec une éventuelle sous-paramétrisation des modèles VAR liée elle-même aux pertes de degré de liberté occasionnées par l'ajout des retards.

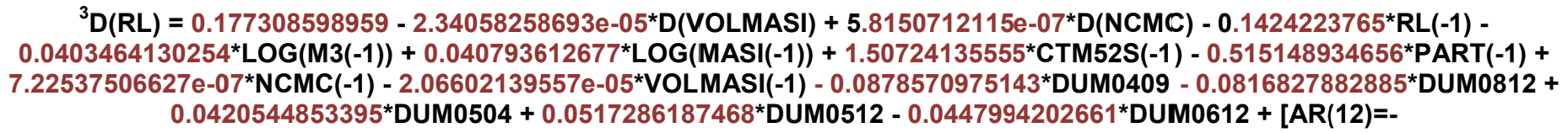

GARCH $=9.70353878082 e-06+0.830539001674^{\star}$ RESID $(-1)^{\wedge} 2+0.235439063788^{\star} G A R C H(-1)$

\section{R-squared $=666621$}

Ainsi, pour un choix de $\mathrm{p}$ retards ${ }^{4}$, correspondant à une autocorrélation d'ordre $\mathrm{p}+1$ des innovations dans une représentation AR (1), les trois modèles utilisés pour développer le test DFA sont les suivants :

$$
\begin{aligned}
& \Delta \mathrm{X}_{\mathrm{t}}=\emptyset \mathrm{X}_{\mathrm{t}-1}+\sum_{\mathrm{i}=1}^{\mathrm{p}} \emptyset_{\mathrm{i}} \Delta \mathrm{X}_{\mathrm{t}-\mathrm{i}}+\varepsilon_{\mathrm{t}} \\
& \Delta \mathrm{X}_{\mathrm{t}}=\emptyset \mathrm{X}_{\mathrm{t}-1}+\sum_{\mathrm{i}=1}^{\mathrm{p}} \emptyset_{\mathrm{i}} \Delta \mathrm{X}_{\mathrm{t}-\mathrm{i}}+\mathrm{c}+\varepsilon_{\mathrm{t}} \\
& \Delta \mathrm{X}_{\mathrm{t}}=\emptyset \mathrm{X}_{\mathrm{t}-1}+\sum_{\mathrm{i}=1}^{\mathrm{p}} \emptyset_{\mathrm{i}} \Delta \mathrm{X}_{\mathrm{t}-\mathrm{i}}+\mathrm{c}+\beta \mathrm{t}+\varepsilon_{\mathrm{t}}
\end{aligned}
$$

Le test de cointégration de JOHANSEN nous éclaire sur le nombre de relation de cointégration et sa forme fonctionnelle en suivant les critères de la trace et valeur propre minimale et les critères d'information d'AKAIKE et de SCHWARZ.

A partir du graphique des résidus on peut valider - visuellement - la qualité de notre estimation, puisque qu'on a pu reproduire une partie considérable de notre série estimée.

\footnotetext{
${ }^{2}$ Lorsqu'il y a des variables dichotomiques dans le modèle, on ne les prend pas en compte dans le test, car elles ne peuvent pas être responsables de l'hétéroscédasticité (car ce sont des variables dont les valeurs ne sont pas liées à la variance de l'erreur).

${ }^{3}$ Le tableau des résultats des estimations sur Eviews en annexes.

${ }^{4}$ Dickey et Fuller ont recours à l'hypothèse de non autocorrélation du terme de l'erreur $(\varepsilon t)$ dans les trois modèles. Mais cette hypothèse risque dans la plupart des cas de ne pas être vraie. Si elle ne tient pas, les valeurs tabulées par Dickey et Fuller ne seront plus correctes. Des retards dans la variable endogène sont alors rajoutés pour tenir compte d'un terme d'erreur autocorrélé.
} 
regroupent les indicateurs de l'économie réelle ${ }^{6}$ à savoir le taux de chômage, le taux de croissance du produit intérieur brut et le taux d'inflation.

\section{Bibliographie}

[1] Bernard A, Tessier J.F., Tessier J.F., Chalifoux D., Actifs réels et placements alternatifs, conférence organisée par Normandin Beaudry, Gestion d'actifs Manuvie et Fiera Axium.

[2] Carbol A., 2006, Gestion de portefeuille, ENSAE.

[3] Christin Y., 2012, Moroccan mutual funds industry.

[4] Cariou T., 2008, Allocation optimale d'actifs sur un portefeuille de responsabilité civile.

[5] Nattahi k., 2013, Hétérogénéité comportementale, mimétisme et dynamique des cours boursiers ? Centre d'économie de l'université Pars Nord, n² 2013-04.

[6] Greene W. H., 2007, Econometric analysis. 6 ed. Upper Saddle River: Prentice Hall.

[7] Droesbeke J.-J., Saporta G., 2010, Analyse statistique des données longitudinales, Technip

[8] Herlemont D., 2014, Econometry of Financial Markets with R-project, YATS Finances \& Technologies.

[9] Le Quéré F., 2010, Gestion déléguée des encours par les investisseurs institutionnels : description et évolution des pratiques, Laboratoire d'économie d'Orléans (LEO), UMR 6221 CNRS, Revue d'économie financière. 